\title{
Internal jugular vein vascular malformation presenting as mass at root of neck: a case report
}

\author{
Prahlad Duggal ${ }^{* 1}$, Pankaj Chaturvedi ${ }^{1}$, Prathamesh S Pai ${ }^{1}$, Deepa Nair ${ }^{1}$, \\ SL Juvekar ${ }^{2}$ and Bharat Rekhi ${ }^{3}$
}

Address: ${ }^{1}$ Head and Neck Services, Department of Surgical Oncology, Tata Memorial Hospital, Parel, Mumbai, India, ${ }^{2}$ Department of Radiodiagnosis, Tata Memorial Hospital, Parel, Mumbai, India and ${ }^{3}$ Department of Pathology, Tata Memorial Hospital, Parel, Mumbai, India

Email: Prahlad Duggal* - duggalprahlad@yahoo.co.in; Pankaj Chaturvedi - chaturvedi.pankaj@gmail.com; Prathamesh S Pai - pspai@gmail.com; Deepa Nair - drdeepanair@hotmail.com; SL Juvekar - sljuvekar@gmail.com; Bharat Rekhi - rekhi.bharat@gmail.com

* Corresponding author

Published: 14 June 2009

BMC Ear, Nose and Throat Disorders 2009, 9:5 doi:10.1/86/1472-68/5-9-5

This article is available from: http://www.biomedcentral.com/l472-68/5/9/5

(C) 2009 Duggal et al; licensee BioMed Central Ltd.

This is an Open Access article distributed under the terms of the Creative Commons Attribution License (http://creativecommons.org/licenses/by/2.0), which permits unrestricted use, distribution, and reproduction in any medium, provided the original work is properly cited.
Received: 8 March 2009

Accepted: 14 June 2009

\begin{abstract}
Background: We report a case of vascular malformation arising from internal jugular vein presenting as mass at root of neck with no clinical stigmata which to the best of our knowledge is the first reported case of an intrinsic vascular malformation arising from the internal jugular vein. Magnetic resonance imaging features of this new entity have been described.

Case presentation: A 27 year male presented with a gradually enlarging, asymptomatic swelling on left supraclavicular region with normal overlying skin. A soft mass, about $7 \times 7 \mathrm{~cm}$ with restricted mobility was found with normal cranial nerve function. Fine needle aspiration cytology showed a hemorrhagic aspirate. Doppler showed a mass displacing left carotid artery posteriorly while left internal jugular vein was not visualized. Magnetic resonance imaging showed a well defined mass isointense to hypointense on TI weighted and hyperintense on T2 weighted and STIR images with fluid-fluid levels. On exploration, a vascular mass arising from left internal jugular vein was found with good tissue planes, which was excised after ligating the patent internal jugular vein above and below the lesion. Histopathologic examination confirmed the diagnosis of vascular malformation.

Conclusion: The diagnosis of intrinsic vascular malformation arising from internal jugular vein should be kept in differential while dealing with masses at root of neck and magnetic resonance imaging features may help in the pre-operative diagnosis of this entity.
\end{abstract}

\section{Background}

Vascular lesions have been classified as hemangiomas or vascular malformations depending on the presence of cellular proliferation [1]. Venous vascular malformations may present as isolated neck masses in adults without the clinical findings typically associated with vascular malformations like skin discolouration, dilated subcutaneous vessels and compressibility [2]. Among the vessels of jugular system in the neck, intrinsic vascular malformations have been described arising from the external jugular vein $[3,4]$. We report a case of intrinsic vascular malformation arising from internal jugular vein presenting as mass at the root of neck with clinical and radiological features suggesting a mass in relation with major vessels of neck. Final 
diagnosis was established only after surgical excision and histopathology. This is the first reported case of an intrinsic vascular malformation arising from the internal jugular vein to the best of our knowledge.

\section{Case presentation}

A 27-year male presented with a gradually enlarging mass in left supraclavicular area for the last three years. There were no associated symptoms. On physical examination, there was a mass about $7 \times 7 \mathrm{~cm}$, soft and non tender on the left side of neck extending from mid neck down to the clavicle. Overlying skin was normal in colour and texture. There were no pulsations or bruit over the mass. Mass showed restricted side to side mobility but was not mobile in supero-inferior direction. There was no other palpable mass or lymph node detected in the rest of the neck. Examination of all the cranial nerves was normal. Doppler of the neck vessels showed that the left common carotid artery was displaced posteriorly by a supraclavicular mass. The mass closely abutted the artery and showed indistinct fat planes with the vessel wall at places but there was no evidence of luminal narrowing noted in the course of carotid artery. The common carotid artery, external and internal arteries showed normal colour, flow and velocity. The left internal jugular vein was not visualized.

Magnetic resonance imaging (MRI) demonstrated a heterogenous well defined mass, $7 \times 4 \times 6 \mathrm{~cm}$ in size in infra hyoid neck on the left side. The lesion appeared isointense to hypointense to muscle on $\mathrm{T} 1$ weighted images [Figure 1] and hyperintense on T2 weighted [Figure 2, 3] and STIR images with fluid-fluid level. The left internal jugular vein superior to the mass was well defined and at the level of the mass was compressed. There was hyperintense signal in the left internal jugular vein secondary to flow related phenomenon. Fine needle aspiration cytology (FNAC) of the mass showed a hemorrhagic aspirate and bleeding from the puncture site stopped with local pressure for few minutes. The patient was planned for exploration and excision of the mass as an elective procedure. Intra-operatively, a mass arising from the lower part of left internal jugular vein was identified which was pushing the common carotid artery and vagus nerve (Figure 4 ). These were dissected off the mass as good tissue planes were available around the mass. The proximal and distal ends of the internal jugular vein were ligated and the mass was completely excised. Gross cut section of the mass showed multiple dilated vascular channels [Figure 5]. Histopathology revealed many dilated, ectatic vascular channels with muscular walls [Figure 6, Figure 7]. The intervening fibroadipose tissue showed scattered chronic inflammatory cells and focal calcification consistent with the diagnosis of vascular malformation.

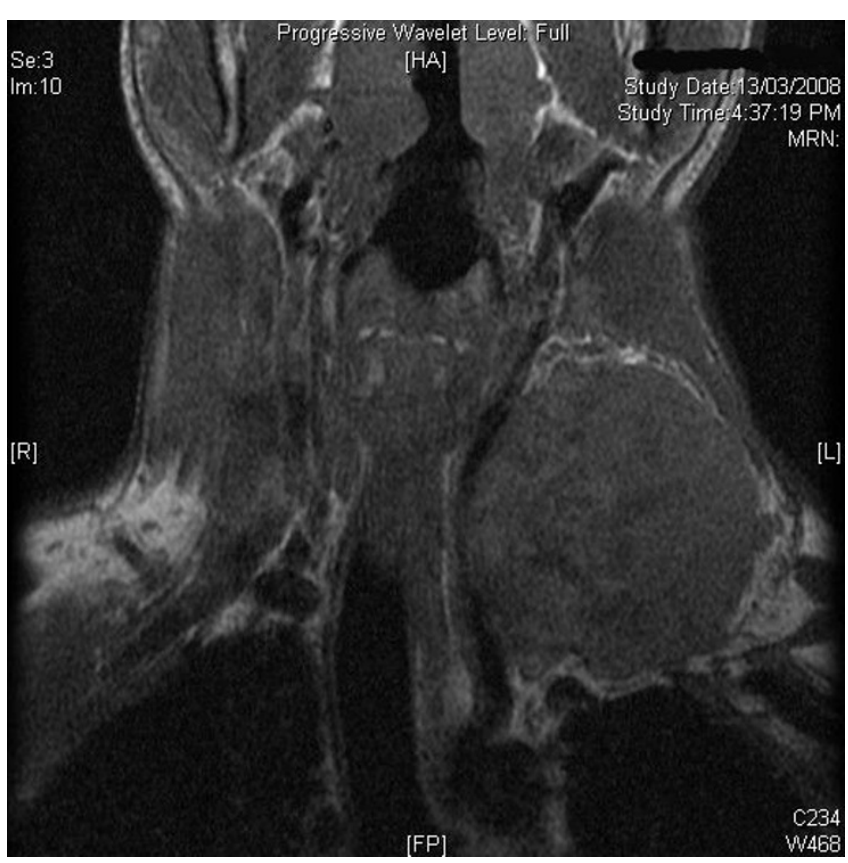

Figure I

TI W magnetic resonance image of the neck (coronal section) showing a mass in infrahyoid neck which is isointense to hypointense to muscle.

\section{Discussion}

Intrinsic vascular malformations arising from the wall of internal jugular vein have not been described in Englishlanguage literature. Few cases of intrinsic vascular malformations arising from external jugular vein have been reported $[3,4]$. We describe a case of vascular malformation of internal jugular vein which presented in an adult as a slowly enlarging mass at the root of neck with no other signs and symptoms, for which the diagnosis was established after surgical exploration and histopathology.

Venous vascular malformations are believed to be present at birth but may not become apparent until later in life. They may expand as a result of trauma, sepsis, hormonal changes or changes in venous pressure $[5,6]$. MR features of head and neck vascular malformations typically show intermediate signal intensity (slightly higher than that of muscles) on $\mathrm{T} 1$ weighted images, hyperintense in $\mathrm{T} 2$ weighted images and variable enhancement after intravenous gadolinium administration [7]. Intrinsic vascular malformations have been reported involving the external jugular vein and having similar features. Authors recommend that this entity involving internal jugular vein should be kept in the differential of such lesions.

Similar MRI characteristics like an isointense T1 signal relative to skeletal muscle with hyperintense and slightly het- 


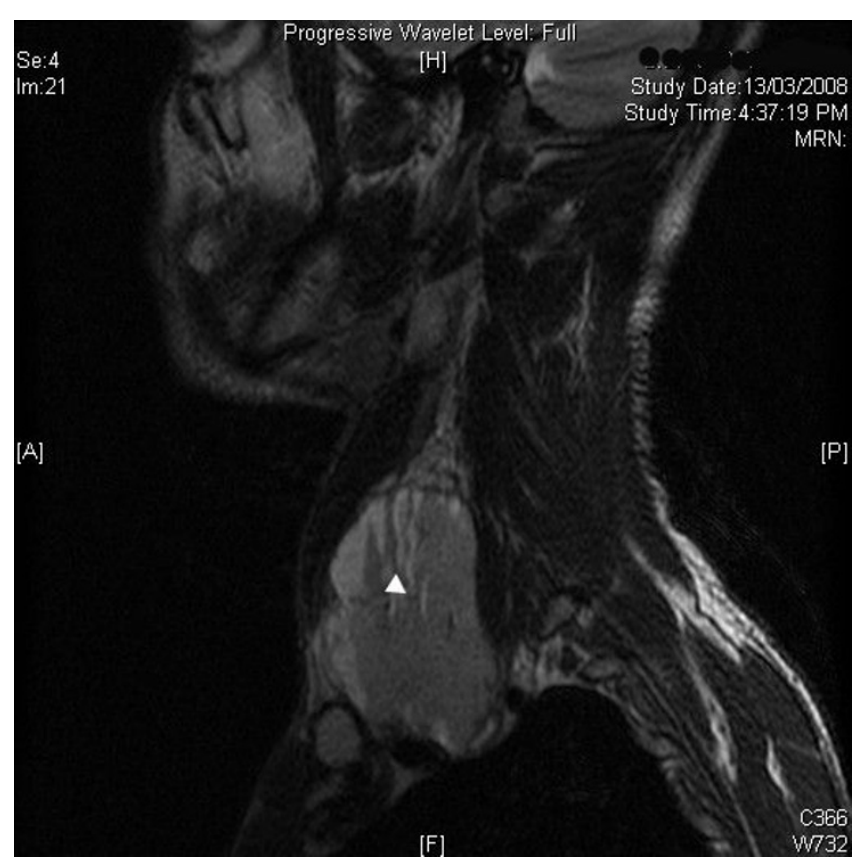

Figure 2

T2 W magnetic resonance image of the neck (sagital section) showing hyperintense mass with fluid-fluid levels (arrow head, pointing upwards).

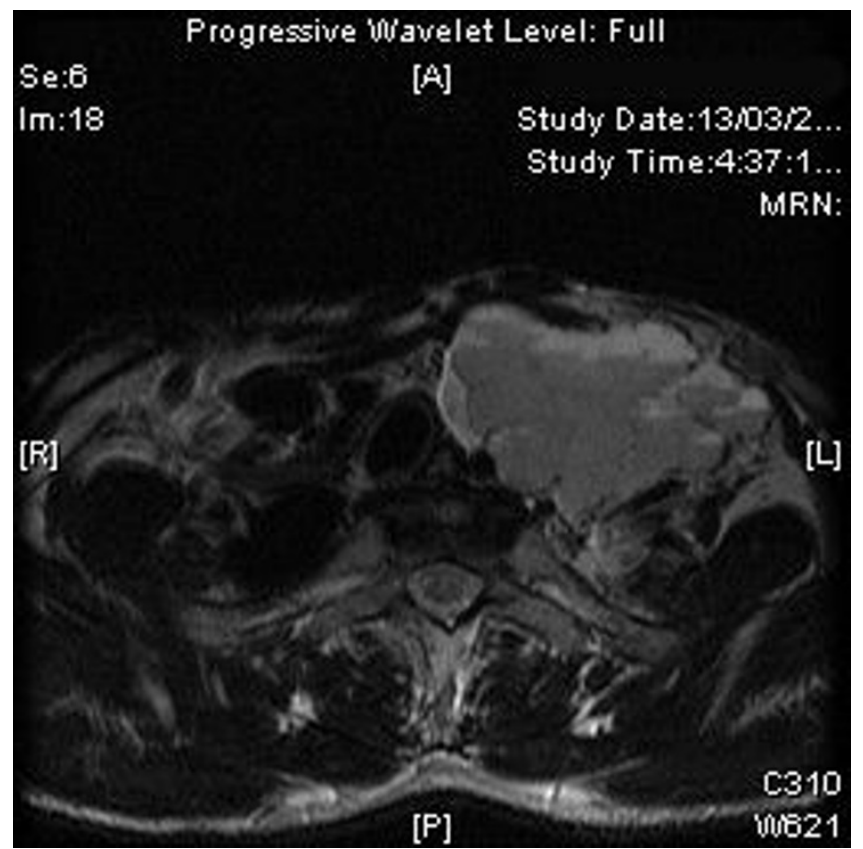

Figure 3

T2 W magnetic resonance image of the neck (axial section) showing hyperintense mass with fluid-fluid levels.

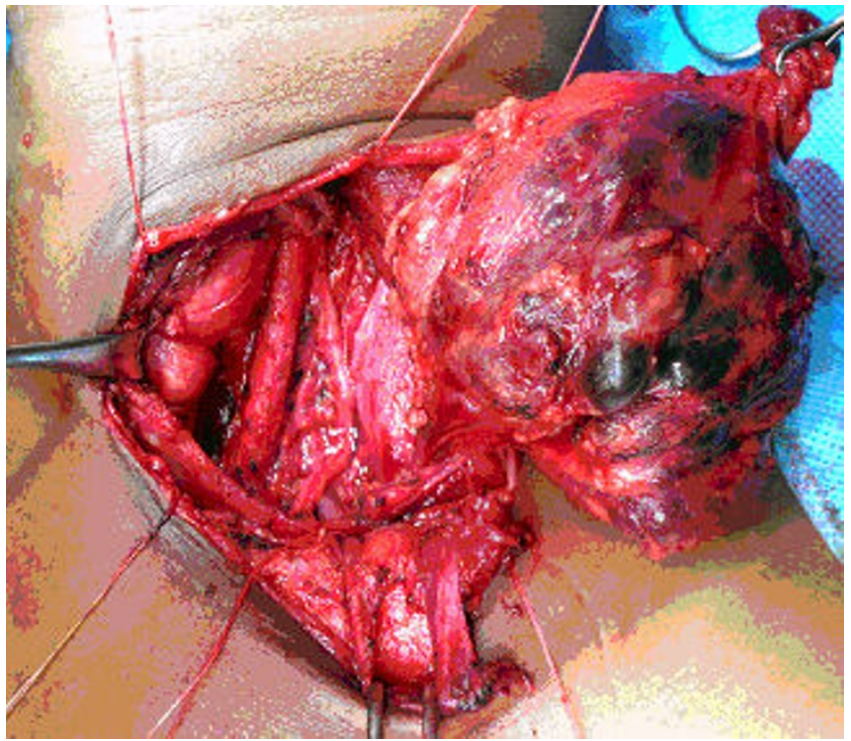

Figure 4

intra-operative photograph showing left internal carotid artery, vagus nerve and vascular mass involving the left internal jugular vein.

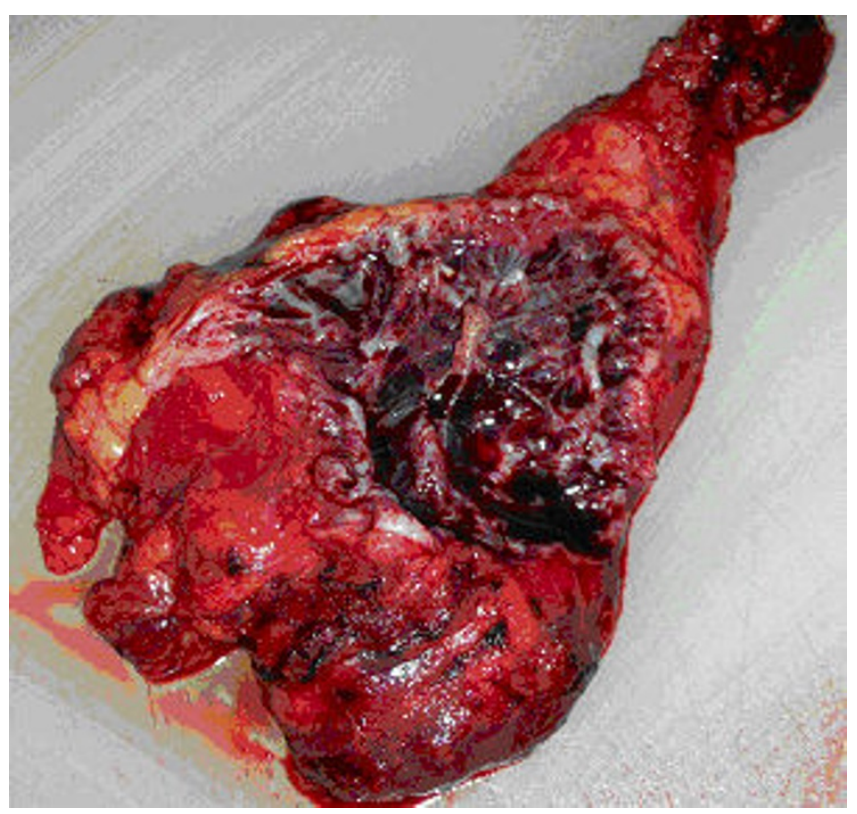

Figure 5

gross cut section of the specimen showing multiple vascular channels. 


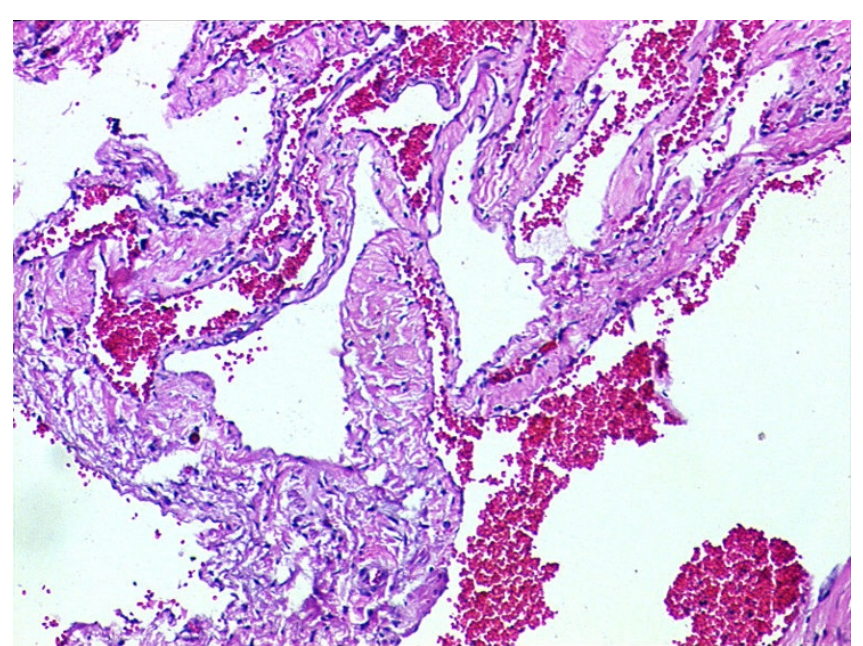

Figure 6

Histological slide of the mass showing dilated vascular channels of vascular caliber with thick muscular walls and focal calcification.

erogenous T2 signal [8] have been reported in head and neck schwannomas. In the present case, the mass was asymptomatic, of long duration with radiological features similar to a cystic schwannoma. Head and neck schwannomas are uncommon nerve sheath neoplasms which may present as diagnostic and management challenges. Majority of extra-cranial schwannomas present as asymptomatic stable neck masses of long standing duration that caused little concern other than the possibility of malignancy and cosmesis [9]. Parapharyngeal space is the most common site for these tumours $[10,11]$ but they can present lower down in the neck [9].

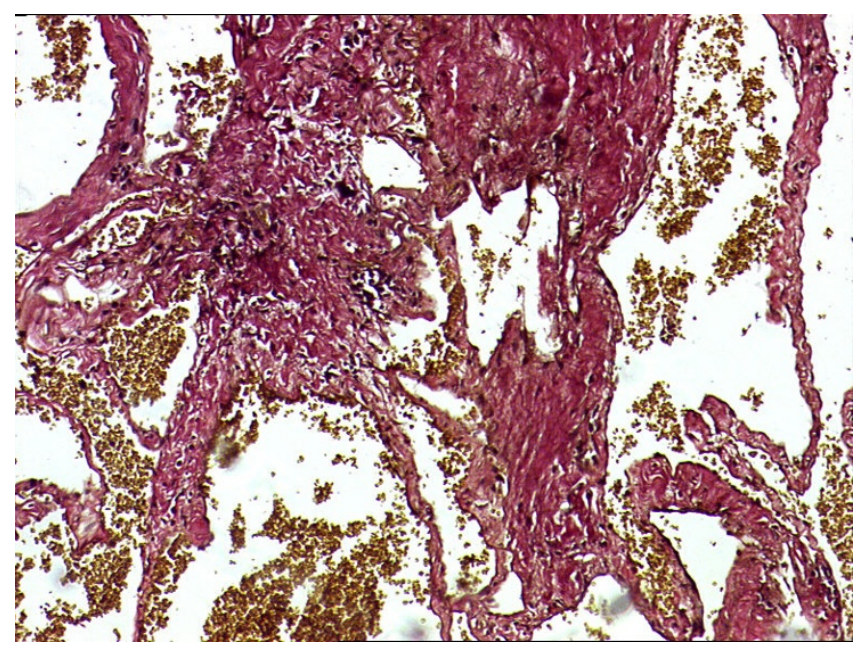

Figure 7

Elastin vangeison (EVG) stained section showing lack of elastic fibers reinforcing venous origin.
The root of the neck is an uncommon location for a soft compressible swelling. Lymph node enlargement can occur in this region but will be firmer in consistency and very well defined. Various swellings, such as intramuscular hemangioma, lymphangioma, lipoma, rhabdomyoma are to be considered in the differential diagnosis[12]. Also, other primary tumours like low-grade malignant hemangioendothelioma, hemangiopericytoma and frankly malignant hemangiosarcoma and leiomyosarcoma may be considered in the differential of these vascular lesions. These tumours are all very rare but may enter the differential list if the lesion shows evidence of enlargement or local infiltration clinically or on imaging studies [4].

In the present case, FNAC was performed pre-operatively but surgical excision rather than a guided biopsy is preferable because of the possibility of extensive bleeding of the punctured vascular lesion [3]. Total excision allows a better histopathologic definition and is curative as has been seen in the present case.

\section{Conclusion}

This is first reported case of an intrinsic vascular malformation of internal jugular vein for which the diagnosis was possible only after surgical excision. This case report underlines the need for head and neck surgeons to be aware of such an entity and should be considered in differential diagnosis for neck masses with similar features.

\section{Competing interests}

The authors declare that they have no competing interests.

\section{Authors' contributions}

DP: was part of surgical team, designed the case report, carried out the literature research and manuscript preparation. CP: was part of the surgical team, guided in drafting the article and revising it critically for important intellectual content. PSP: carried out the literature research, manuscript editing and manuscript review. ND: was part of the surgical team, carried out the manuscript editing and manuscript review. JSL: helped in the radiological aspects of the present case and carried out the manuscript editing. $\mathrm{RB}$ : helped in the pathological aspects of the present case and carried out the manuscript editing. All the authors read and approved the final manuscript.

\section{Acknowledgements}

Written consent was obtained from the patient for publication of the patient's details.

\section{References}

I. Mulliken JB, Glowacki J: Hemangiomas and vascular malformation in infants and children: a classification based on endothelial characteristics. Plast Reconstr Surg 1982, 69:412-422. 
2. Martin JF, Roy AH, Roland JT: Clinically unsuspected venous malformations limited to the submandibular triangle: $\mathbf{C} \mathbf{T}$ findings. Am J Neuroradiol 1995, 16:491-494.

3. Sarteschi LM, Bonanomi G, Mosca F, Ferrari M: External jugular vein hemangioma occurring as a lateral neck mass. J Ultrasound Med 1999, 18:719-72I.

4. Ahuja AT, Yeun H, Wong K, King AD, Abdullah V, To E, Chau Y, Ma $K$ : External jugular vein vascular malformation: sonographic and MR imaging appearences. Am J Neuroradiol 2004 25:338-342.

5. Finn MC, Glowacki J, Mulliken JB: Congenital vascular lesions: clinical applications of a new classification. J Pediatr Surg 2004, 18(6):894-900.

6. Berenstein A, Lasjaunias P: Surgical neuroangiography Volume 4. Berlin: Springer Verlag; 1992:387-397.

7. Fordham LA, Chung CJ, Donnelly LF: Imaging of congenital vascular and lymphatic anomalies of the head and neck. Neuroimaging Clin N Am 2000, I (1): I 17-136.

8. Beaman FD, Kransdrof MJ, Menke DM: Schwannoma: radiologicpathologic correlation. Radiographics 2004, 24: I477-I48I.

9. Kang Gavin CW, Soo Khee Chee, Lim Dennis TH: Extracranial non-vestibular head and neck schwannomas: a ten year experience. Ann Acad Med Singapore 2007, 36:233-240.

10. Leu YS, Chang KC: Extracranial head and neck schwannomas: a review of 8 years experience. Acta Otolaryngol 2002, I 22:435-437.

II. Malone JP, Lee WJ, Levin RJ: Clinical characteristics and treatment outcome for nonvestibular schwannomas of the head and neck. Am J Otolaryngol 2005, 26: I08-I I2.

12. Panda NK, Reddy CEE, Sharma SC, Powari M: Intramuscular hemangioma at the root of neck. The Journal of Otolaryngology 2003, 32(3):206-209.

\section{Pre-publication history}

The pre-publication history for this paper can be accessed here:

http://www.biomedcentral.com/1472-6815/9/5/prepub

Publish with Biomed Central and every scientist can read your work free of charge

"BioMed Central will be the most significant development for disseminating the results of biomedical research in our lifetime. "

Sir Paul Nurse, Cancer Research UK

Your research papers will be:

- available free of charge to the entire biomedical community

- peer reviewed and published immediately upon acceptance

- cited in PubMed and archived on PubMed Central

- yours - you keep the copyright 